Research Article

\title{
The Evaluation Model of Network QoS Based on Intelligent Water Droplets Algorithm
}

\author{
Fang Li $\mathbb{D}^{1},{ }^{1}$ Yunlan Wang, ${ }^{1}$ and Peng $\mathrm{Li}^{2}$ \\ ${ }^{1}$ Yiwu Industrial \& Commercial College, Yiwu 322000, Zhejiang, China \\ ${ }^{2}$ Hunan University of Science and Technology, Xiangtan 411201, Hunan, China \\ Correspondence should be addressed to Fang Li; tyrone889@protonmail.com
}

Received 21 January 2020; Revised 23 March 2020; Accepted 27 March 2020; Published 8 June 2020

Academic Editor: Stefan Balint

Copyright $\odot 2020$ Fang Li et al. This is an open access article distributed under the Creative Commons Attribution License, which permits unrestricted use, distribution, and reproduction in any medium, provided the original work is properly cited.

In order to solve the problem of the user demands to the quality of service (QoS) of network, this paper provides a novel QoS evaluation model based on the intelligent water droplets (IWD) algorithm. The model firstly gives the evaluation indexes of network QoS and establishes a multiobjective optimization function for solution in combination with the improved IWD. Then, mathematical simulation is used to conduct the in-depth study of the key factors influencing the evaluation model. Compared with the other methods, the algorithm has shown better adaptability in the simulation results.

\section{Introduction}

Web services with the same or similar functions continue to emerge with its increasing number. Although web service composition technology can find service combinations that meet user needs, the combinations are with similar functions and lacks evaluation parameters other than functional attributes, so it is difficult to choose the best service from them. The way, to efficiently implement service selection and combination among different quality of service (QoS) candidate service sets, has become a key link in building a highly reliable and high-quality combined service. QoSbased Web service recommendation can be divided into two categories [1-7]: one is to model the service selection with multiple QoS constraints as a directed acyclic graph optimal path problem and give a service selection algorithm based on this model; the other is to establish QoS ontology with the description and deduction ability of which the accuracy of service matching can be improved.

A large amount of evaluation indexes are proposed for the network QoS. Wang [8] proposed a typical QoS-aware web service orchestration engine and used a three-layered pyramidal structure to capture the requirements of the customers, characteristics, and behaviors of engine. Tran et al. [9] proposed a novel approach for designing and developing a QoS ontology and its QoS-based ranking algorithm for evaluating web services. The QoS ontology could support not only describing QoS information in great detail but also facilitating various service participants expressing their QoS offers and demands at different levels of expectation. Luo et al. [10] presented a novel QoS prediction approach to serve this purpose through the combination of fuzzy neural networks and adaptive dynamic programming. The experimental results on a large-scale QoS service dataset verified the prediction accuracy of this approach. Chen et al. [11] have studied QoS dependency-aware service composition considering multiple QoS attributes, and it takes advantage of vector ordinal optimization techniques to search for Pareto optimal composition solutions with QoS dependency involved. Luo et al. [12] presented a data-driven scheme of predicting the missing QoS values for the IIoT based on a kernel least mean square algorithm, in which kernel least mean square algorithm was used to analyze the hidden relationships between all the known QoS data and corresponding QoS data with the highest similarities. Parejo et al. [13] proposed a metaheuristic algorithm for performing QoS-aware web service composition at runtime. Mao et al. [14] proposed a search-based prediction framework to address the QoS ranking problem, and a fitness function for an ordered service sequence is defined to guide 
search algorithm to find high-quality ranking results. Ding et al. [15] addressed the issue of selecting and composing web services via a genetic algorithm and gave a transaction and QoS-aware selection approach. Temglit et al. [16] proposed a distributed and optimal QoS selection approach based on multiagents paradigm and distributed constraint optimization formalism, and it takes into account the specificities of the service composition context and the satisfaction of the global user's constraints. Gu et al. [17] proposed an agent-based distributed QoS multicast routing algorithm, and it takes advantage of the cooperation of different agent groups to find the optimum multicast routing that satisfies the QoS constraint. Huang et al. [18] presented an efficient service selection scheme to help service requesters select services by considering two different contexts: single QoS-based service discovery and QoS-based optimization of service composition. Lo et al. [19] presented a local neighborhood matrix factorization application that incorporates domain knowledge in artificial intelligence technique, and it integrated the geographical information to build up an extended matrix factorization approach for personalized QoS prediction. However, most of the existing evaluation methods for the QoS are based on describable quality attributes or reputable recommendation information by default, so the evaluation results may become inaccurate.

In order to recommend high-quality services to users, a key issue is how to obtain quality parameter values for web services, which are generally represented by QoS. However, in practice, the QoS of most web services is unknown to users. In addition, the QoS parameters of web services are affected by factors such as the network environment. As a result, users at different locations may observe different QoS values on the same service. Although the user can call the web service to evaluate its own QoS, it is not realistic to accurately evaluate the QoS of a large number of candidate services in a short time since the users are not experts in evaluating the service.

With the studies above, in order to further fulfill user demands on network QoS, the paper provides a novel QoS evaluation method on the basis of improved intelligent water droplets (IWD). The IWD, with high self-organization, is a novel algorithm merging probability choice and heuristic search and very adaptive to complex problems. This paper considers the number of users on the web as that of water droplets, and the drop rolling rule is regarded as the evaluation index of service quality. The process of water droplets optimization is considered as that of service quality optimization and the group that has used the service is considered as the sediment taken away. The best web service can be found out with the continuous iteration and update therefrom. The paper provides the evaluation indexes for the network QoS and then establishes a multiobjective optimization function to solve the problem with the IWD.

\section{Evaluation Indexes on Web Services}

Because there are a large number of candidate web services, it is not feasible for users to experience all services and then make a decision. Service recommendations can be made by referring to the QoS history of other users, while most existing service recommendation methods use the QoS history record of all users. Due to the large amount of QoS data, the time complexity of service recommendation is exorbitant and the real-time capability is not strong. Moreover, different users have different interests that may make users not to access some certain services, which may just meet the user's requirements for QoS performance, resulting in a decrease in the accuracy of the recommendation. Although web service composition technology can find service combinations that meet user needs, the result is a batch of service combinations with similar functions. In addition, there is a lack of evaluation parameters other than functional attributes, so it is difficult to choose the best service, too. To solve this problem, a web service quality composition method based on nonfunctional attributes is proposed.

The evaluation of network QoS, adopting specified tools and methods, is an active procedure for the objective evaluation and measurement of the QoS, according to certain standards and rules. Many important attributes can be used to measure the network QoS, like the latency, the accuracy and the reliability. The quality vector of a certain web service $w$ is defined as $Q(w)$ herein. When the service is being used, the QoS may be different from the predefined value, and the user may consider QoS quite poor because of the large difference. In order to analyze the deviation between the actual value and the predictive value of QoS, the paper defines the evaluation indexes for the network QoS. For web service $w$, making the predefined quality vector be $Q(w)=\left(Q_{1}, Q_{2}, \ldots, Q_{n}\right)$ and the actual quality vector be $Q^{\prime}(w)=\left(Q_{1}^{\prime}, Q_{2}^{\prime}, \ldots, Q_{n}^{\prime}\right)$, the evaluation value of the network QoS will be

$$
P(w)=1+\frac{1}{n}\left(\left|\sum_{i=1}^{n} \frac{Q_{i}-Q_{i}^{\prime}}{1+Q_{i}}\right|-\left|\sum_{i=1}^{n} \frac{Q_{i}^{\prime}-Q_{i}}{1+Q_{i}^{\prime}}\right|\right) .
$$

If $P(w) \geq 1$, the actual QoS is or better than the predefined one; if $P(w)<1$, the QoS is poor; if $P(w)<0$, the web service is nearly unavailable. For the service $w$, users can select different types of it based on the historical evaluation, which can be set as the recommendation reliability $\xi i$ of the service $w$; for service $w$, making $p_{i}^{w(k)}$ be the satisfaction evaluation to the quality attribute $q \in Q w(Q w$ is the set of the quality attributes of the service $w$ ) of web service $w$ by the user $i$, and $Y w$ be the set of user group who have used service $w$; the satisfaction of user $i$ and the user group $Y w$ to certain quality attribute $k$ of the service $w$ is

$$
\phi_{i, Y w}^{w(k)}=1-\sum_{y}|Y w|^{-1} p_{y}^{w(k)}
$$

Therefore, the satisfaction to the service $w$ can be expressed by

$$
\Phi_{i, Y w}^{w}=|K|^{-1} \sum_{k}^{K} \phi_{i, Y w}^{w(k)}
$$

where $|K|$ is the number of the quality attributes in service, so the reliability of the recommended service $w$ is 


$$
\xi_{i}=|\chi|^{-1} \sum_{w}\left(1-\Phi_{1, Y_{w}}^{w}\right),
$$

where $\chi$ is the set of the services commented by user $i$.

In actual web service, due to different experiences and environments of users, it is not enough to consider solely the reliability of the recommendation. Therefore, the paper takes the preference similarity into consideration, and the preference similarity of users $i$ and $j$ in service $w$ is

$$
\psi_{i, j}^{w}=1-\left(\frac{1}{2|K|} \sum_{k=1}^{|K|}\left|q_{i, w}^{k}-q_{j, w}^{k}\right|\right)^{(1 / 2)},
$$

where $q_{i, w}^{k}=\left\{q_{i, w}^{1}, q_{i, w}^{2}, \ldots, q_{i, w}^{k}, \ldots, q_{i, w}^{n}\right\}$ is the evaluation set of all attributes of service $w$ given by user $i$ after using service, and similarly, $q_{j, w}^{k}=\left\{q_{j, w}^{1}, q_{j, w}^{2}, \ldots, q_{j, w}^{k}, \ldots, q_{j, w}^{n}\right\}$ is the evaluation set of all attributes of service $w$ given by user $j$.

Setting user $i$ as the service applicant and user $j$ as the service recommender who used service $w$ before, the evaluation results of the service $w$ by the user $i$ are

$$
\mu_{i, j}^{w}=\frac{\sum_{k}\left(\psi_{i, j}^{k} q_{i, w}^{k}\right)}{\sum_{k} \psi_{i, j}^{k}} .
$$

Meanwhile, mean absolute deviation (MAE) is used to represent the reliability; the smaller the MAE, the higher the QoS. MAE is as follows:

$$
\text { MAE }=\frac{1}{|N|} \sum_{i}\left|p_{i}-q_{i}\right| \times 100 \%,
$$

where $p_{i}=\left\{p_{1}, p_{2}, \ldots, p_{N}\right\}$ represents the evaluation set of the users and $q_{i}=\left\{q_{1}, q_{2}, \ldots, q_{N}\right\}$ represents the actual score set.

In combination with the satisfaction $\phi$, the reliability $\xi$, the preference similarity $\psi$, and the MAE, the objective optimization model is defined as follows:

Objective function:

$$
z=\min \left(\left(\sum_{i} \frac{1}{\alpha \phi_{i, Y w}^{w(k)}+\beta \xi_{i}+\gamma \psi_{i, j}^{k}}+\lambda \mathrm{MAE}\right)\right) .
$$

Constraint conditions:

$$
\begin{aligned}
& \psi_{i, j}^{w}= \begin{cases}\frac{1}{|S|} \sum_{k=1}^{|S|} \psi_{(i, j)}^{k}, & |S|>\eta, \\
\Phi_{i, Y w}^{w}=|K|^{-1} \sum_{k} \phi_{i, Y w}^{w(k)}, \text { others, }\end{cases} \\
& \mu_{i, j}^{w}=\frac{\sum_{k=1}^{Y w}\left(\psi_{i, j}^{k} q_{i, w}^{k} \delta i, w\right)}{\sum_{k=1}^{Y w}\left(\psi_{i, j}^{k} \delta i, w\right)},
\end{aligned}
$$

$\alpha+\beta+\gamma+\lambda=1,0<\alpha<1,0<\beta<1,0<\gamma<1,0<\lambda<1$,

$\alpha \phi+\beta \xi+\gamma \psi>0$,

$\operatorname{MAE} \geq 0$, where the objective function $z$ is used to obtain the optimal QoS of web, and $\alpha, \beta, \gamma$, and $\lambda$ are weight coefficients. According to the services in the set $S$, the preference similarities of users $i$ and $j$ in each service are synthesized, $\eta$ is set to be the threshold value of set S, and formula (9) stands for the preference similarity. When the recommendation reliability is lower than $\rho$, the system will automatically ignore the service and only adopt reputable users' comments in the evaluation of QoS attributes. The click rate of each service determines users' knowledge to it, so the paper uses the factor $\delta(i, w)=\operatorname{sum} i w / \sum_{N}$ sum $j w$ to modify the evaluation value; in the formula, sumiw is the service $w$ used by user $i$ and $Y_{W}$ is the number of users who used the service $w$, and formula (10) represents the modified QoS attribute.

\section{Solution Algorithm}

The objective function established above is a nonlinear programming problem, and its optimal solution is usually obtained through rapid convergence by using artificial intelligence algorithm. By imitating the natural process of water droplets dripping, the IWD has the advantage of solving nonlinear problems quickly.

Therefore, this paper improves the intelligent water droplet algorithm for the objective function mentioned above. The improved algorithm maintains the diversity of the population through mutation and exchange methods and adopts the optimal selection method to accelerate convergence and improve the speed as well as accuracy of service optimization problems at the same time. The IWD belongs to successive searching algorithm, of which the basic idea is, by dividing paths, the water droplets move in dispersed steps. The water droplets have two attributes of movement velocity $v^{\mathrm{IWD}}$ and sediment content $s^{\mathrm{IWD}}$, which are updated with the position change of the water droplets; the IWD has the main characteristic of evaluating the minimum value of the divided paths.

Corresponding to the abovementioned objective function of web service quality, this paper considers the number of users in the web as that of water droplets, the path of water droplet seeking segmentation as that for finding services in the network, the speed of motion as that of users finding services, and the process of water droplets optimization as that of service quality optimization. In addition, the rule of water droplets rolling down is regarded as an evaluation index of service quality. The groups who have used the service are regarded as the sediment taken away, and each water drop is regarded as a different service in the web service. Each optimization cycle filters a service that carries the most sediment and iterates through this to find the best service in the web service.

The solution steps are as follows:

Step 1: initialized: making the number of users be $N$, velocity update be coefficient $\partial$, the searching velocity of user be $v^{\mathrm{IWD}}$, the number of users who have used the service be $s^{\mathrm{IWD}}$, the maximum number of iterations be Max, and the number of optional service path be $k$. 
Step 2: on the basis of the initialized parameters, the satisfaction $\phi$, the reliability $\xi$, the preference similarity $\psi$, and the MAE, the adaptability of QoS objective function is calculated:

$$
f=\frac{k}{1+e^{z}}
$$

where $k$ stands for a constant, $k>0$, and the minimum value of the adaptability in the first iteration is stored in the array A[]; the optimal solution of each iteration is expressed by OPT, so as to evaluate all service paths.

Step 3: making the probability of a user moving from service $i$ to service $j$ be $p_{i, j}$, its velocity increment $\Delta v^{\text {IWD }}$ and the number of users who have used the service $s^{\mathrm{IWD}}$ are in nonlinear inverse proportion: $\Delta v^{\mathrm{IWD}} \infty^{\mathrm{NL}}\left(1 / s_{i, j}^{\mathrm{IWD}}\right)$,. Therefore, the searching velocity of the users meets

$$
\begin{aligned}
v^{\mathrm{IWD}} & =v_{0}^{\mathrm{IWD}}+\Delta v^{\mathrm{IWD}}, \\
\Delta v^{\mathrm{IWD}}(t) & =\frac{a^{\prime}}{b^{\prime}+c^{\prime} s_{i, j}^{2 \partial}},
\end{aligned}
$$

where $a^{\prime}, b^{\prime}, c^{\prime}$, and $\partial$ are weight parameters.

Step 4: making the number of users who have used the service meet $s^{\mathrm{IWD}}=s^{\mathrm{IWD}}+\Delta s^{\mathrm{IWD}}$; the reduced number of groups is

$$
\begin{aligned}
\Delta s_{i, j} & =\Delta s^{\mathrm{IWD}}=\frac{a}{b+c \tau_{i, j}^{v^{\mathrm{IWD}}}}, \\
\tau_{i, j}^{v^{\mathrm{IWD}}} & =\frac{\mathrm{HUD}_{i, j}}{v^{\mathrm{IWD}}}
\end{aligned}
$$

where $a, b$, and $c$ are constants other than 0 and the $\tau_{i, j}^{\nu^{\mathrm{IWD}}}$ is the time spent by the user moving from service $i$ to service $j$ at the velocity $v^{\mathrm{IWD}}$; HUD is an inverse heuristic function, representing the extent to which users refuse to use other services, which is in direct proportion with the distance between the service $i$ and the service $j$, so the number of users from service $i$ to service $j$ is

$$
s_{i, j}=\mu s_{i, j}-\omega \Delta s_{i, j}
$$

where $\omega+\mu=1$, and both $\omega$ and $\mu$ are decimals in $(0,1)$. Step 5: for the multiple paths to find a service, the user often chooses a path with smaller groups. Therefore, the probability of the user from service $i$ to service $j$ is $p_{i, j}=\left(f\left(s_{i, j}\right) / \sum f\left(s_{i, k}\right)\right)$, and $\sigma$ is an optional smaller integer to ensure that $f\left(s_{i, j}\right)$ is an effective value:

$$
\begin{aligned}
& f\left(s_{i, j}\right)=\frac{1}{\sigma+g\left(s_{i, j}\right)}, \\
& g\left(s_{i, j}\right)=\left\{\begin{array}{l}
s_{i, j} \min \left(s_{i, j} \geq 0\right), \\
s_{i, j}-\min \left(s_{i, j}\right) \min \left(s_{i, j}\right)<0,
\end{array}\right.
\end{aligned}
$$

where $g\left(s_{i, j}\right)$ is to make sure that the number of groups between the service $i$ to service $j$ is not negative and $\min \left(s_{i, j}\right)$ stands for the minimum number of groups from service $i$ to service $j$.

Step 6: the velocity and the number of groups are updated, and mutation operation is conducted to each user:

$$
\left\{\begin{array}{l}
v_{i, j}=p_{i, j} v_{i, j}+\operatorname{rand} v_{i, j}, \\
s_{i, j}=p_{i, j} s_{i, j}+(1-\text { rand }) s_{i, j} .
\end{array}\right.
$$

Step 7: according to formula (23), interlace operation is conducted to the user meeting certain conditions with the optimal individuals obtained at the first time; meanwhile, the number of users seeking service path is constantly updated, which is more conducive to avoiding local optimum:

$$
\left\{\begin{array}{l}
v_{i, j}=p_{i, j} v_{i, j}+e^{1-f}(1-\text { rand }), \\
s_{i, j}=p_{i, j}\left(s_{i, j}-\min \left(s_{i, j}\right)\right)+e^{f} \operatorname{rand}() .
\end{array}\right.
$$

Step 8: to determine whether the current number of iterations reaches the maximum value $M a x$, if so, the A [] and the optimal solution OPT are output. Otherwise, the procedure goes back to Step 3 for repetition.

Step 9: the end.

\section{Numerical Analysis}

To verify the effectiveness of the improved IWD in QoS evaluation, the paper conducts a simulation experiment. Firstly, relevant parameters are set as follows: maximum number of iterations $\operatorname{Max}=100, \partial=0.05, a=0.6, b=1.2$, $c=1.4, a^{\prime}=0.5, b^{\prime}=1.5$, and $c^{\prime}=1.2$. The number of users who used the service $w: Y w=120$, and the threshold value of the recommendation reliability $\rho=1$. The number of water droplets $N=100$, and the initial sediment content and the initial velocity are 0 . In Figure 1 , the paper compares the MAEs change of the three algorithms (BA, POS, and IWD) along with the change of the reliable recommendation number, and it is can be seen from the figure that the IWD has a great advantage that the MAE of IWD is smaller than that of the other two algorithms regardless of the reliable recommendation number. As mentioned before, the smaller the MAE value is, the better the recommendation reliability 


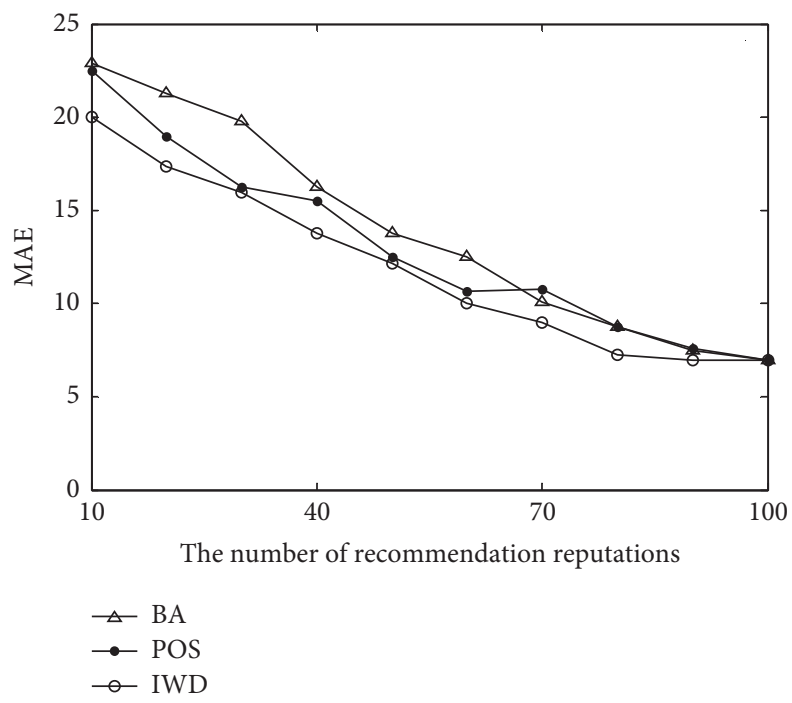

FIgURE 1: The changing of MAE

TABLE 1: Statistical results.

\begin{tabular}{lcccc}
\hline Algorithm type & Times of running & Times of optimal solution found & Mean value & Worst value \\
\hline BA & 100 & 54 & 79 & 69 \\
POS & 100 & 62 & 88 & 71 \\
IMD & 100 & 94 & 96 & 86 \\
\hline
\end{tabular}

of the service will be, proving that the quality of the service is better than that of others. Of course, the figure also reflects that the MAE of the service is reducing along with the increase of the reliable recommendation number, showing that the recommendation reliability of the service is increasing. Meanwhile, each of the three algorithms of BA, POS, and IWD is used to calculate for 100 times, respectively, in this paper to count how many times each algorithm can find the optimal solution as well as the average value and the worst value of the evaluation service quality, as shown in Table 1. It is shown that the optimal solution times and the evaluation values of the QoS in different algorithms are greatly different. Among the three algorithms, IWD has better performance and can effectively find the web service with the best quality.

Meanwhile, Figure 2 shows the variation of the number of optimal solutions in IWD along with the iteration, and it can be seen that the number of the optimal solutions increases with the increase of iteration. In addition, Figure 3 shows that the convergence time also increase along with the iteration increase. Therefore, the convergence time and the number of the optimal solutions need to be considered in a comprehensive manner, which needs repeated experiments to find an equilibrium point.

In Figure 4, the paper compares the convergence precisions of the iteration in BA, POS, and IWD algorithms, and it is shown that the convergence precision of the algorithms reduces along with the iteration increase; even so, the convergence precision of IWD is higher than that of the other two algorithms. From what has been discussed above, it can be concluded that the IWD has a great advantage in

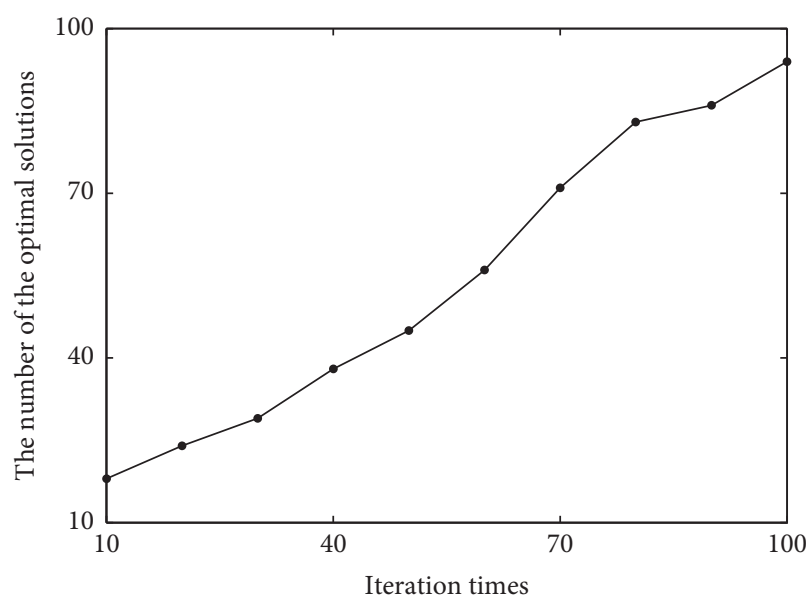

Figure 2: The change of the optimal solutions.

evaluating the network QoS and is capable of recommending web service with better quality.

Figure 5 illustrates QoS evaluation results along with the iteration change in the IWD; it can be seen that the iteration times can greatly influence the QoS and their relationship is nonlinear. When the iteration value is between 40 and 50, it is clear enough to see that the QoS achieves a peak value. However, the QoS is not always increasing along with the increase of the iteration, which can be influenced by other factors. Therefore, comprehensive consideration of different factors is required in QoS selection. 


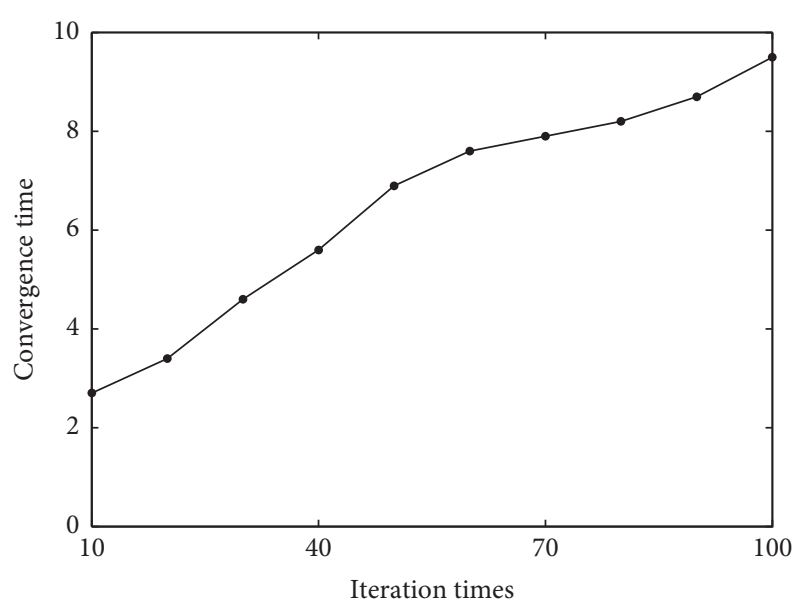

FIgURE 3: The change of convergence time.

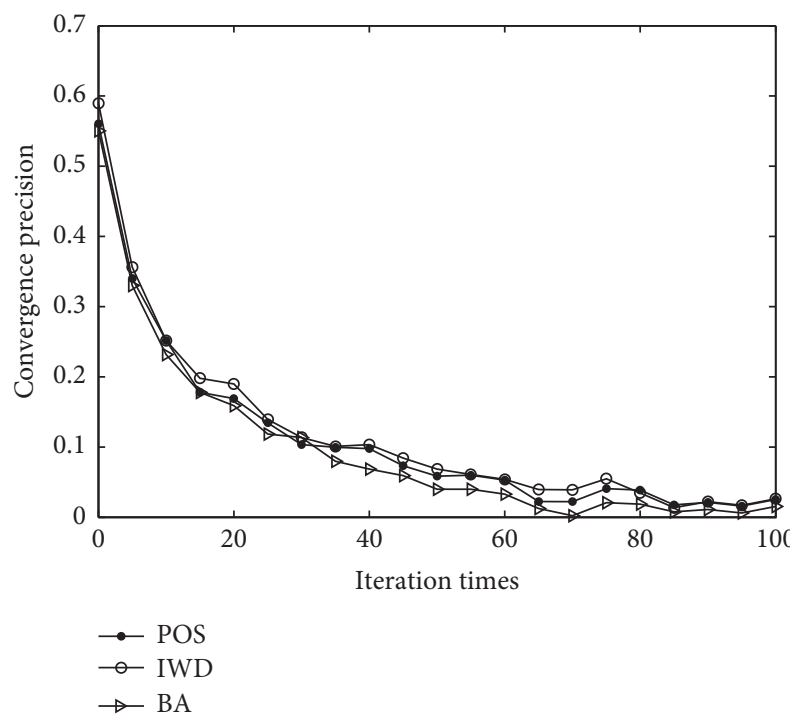

Figure 4: Comparison of convergence precision.

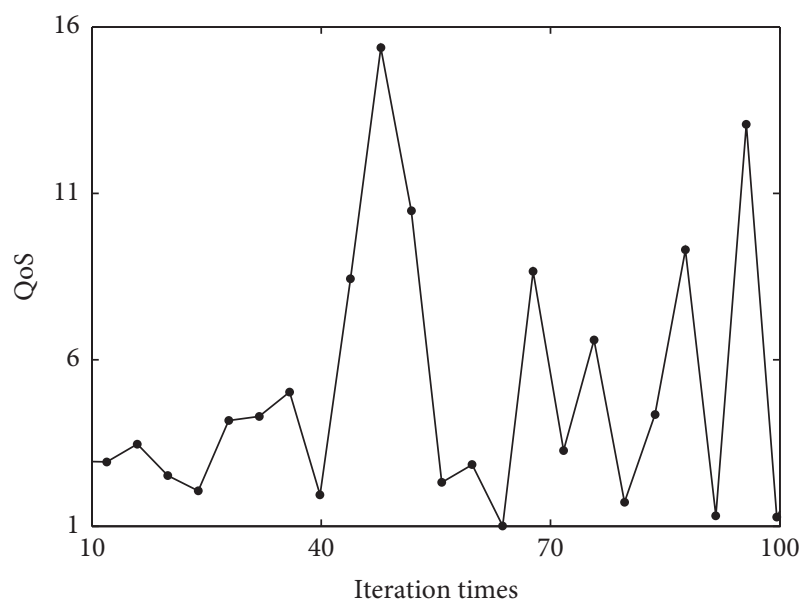

FIGURE 5: QoS evaluation results.

\section{Conclusions}

Users can obtain their accurate QoS historical data in a real runtime environment by using a web service. Thus, by introducing the QoS history of the service into the web service recommendation, the accuracy of service recommendation can be improved. Since the network QoS cannot meet users' demands in many aspects, this paper provides a QoS evaluation method based on the IWD. IWD is a novel algorithm in the field of swarm intelligence algorithm, simulating the process by which the natural environment interacts with the water flow to form a river channel and can be used to solve some complex scientific problems. The service quality evaluation model is established by combining the service evaluation index system with the mathematical model of intelligent water droplets algorithm. The experimental results also prove the feasibility of the method, and in the future study, on the web service, a complete evaluation system can be established by referring to this paper.

\section{Data Availability}

The data used to support the findings of this study are included within the article.

\section{Conflicts of Interest}

The authors declare that they have no conflicts of interest.

\section{Acknowledgments}

This work was supported by the Scientific Research Project Foundation of Yiwu Industrial and Commercial College (No. ZD2020JD122-01).

\section{References}

[1] Y.-Y. Fanjiang, Y. Syu, J.-Y. Kuo, and Y. K. Jong, "Search based approach to forecasting QoS attributes of web services using genetic programming," Information and Software Technology, vol. 80, pp. 158-174, 2016.

[2] Y. Xu, J. Yin, S. Deng, N. N. Xiong, and J. Huang, "Contextaware QoS prediction for web service recommendation and selection," Expert Systems with Applications, vol. 53, pp. 7586, 2016.

[3] K. Farsandaj and C. Ding, "Scatter/gather browsing of web service QoS data," Future Generation Computer Systems, vol. 28, no. 7, pp. 1145-1154, 2012.

[4] Z. Ye, S. Mistry, A. Bouguettaya, and H. Dong, "Long-term QoS-aware cloud service composition using multivariate time series analysis," IEEE Transactions on Services Computing, vol. 9, no. 3, pp. 382-393, 2016.

[5] Y.-M. Kim, E.-J. Lee, and H.-S. Park, "Ant colony optimization based self-organizing QoS framework in IP networks," IEEE Communications Letters, vol. 14, no. 11, pp. 1074-1076, 2010.

[6] S. K. Gavvala, C. Jatoth, G. R. Gangadharan, and R. Buyya, "QoS-aware cloud service composition using eagle strategy," Future Generation Computer Systems, vol. 90, pp. 273-290, 2019.

[7] B. K. J. Al-Shammari, N. Al-Aboody, H. Al-Raweshidy, and S. Al-Raweshidy, "IoT traffic management and integration in 
the QoS supported network," IEEE Internet of Things Journal, vol. 5, no. 1, pp. 352-370, 2018.

[8] Y. Wang, "A formal model of QoS-aware web service orchestration engine," IEEE Transactions on Network and Service Management, vol. 13, no. 1, pp. 113-125, 2016.

[9] V.X. Tran, H. Tsuji, and R. Masuda, "A new QoS ontology and its QoS-based ranking algorithm for Web services," Simulation Modelling Practice and Theory, vol. 17, no. 8, pp. 13781398, 2009.

[10] X. Luo, Y. Lv, R. Li, and Y. Chen, "Web service QoS prediction based on adaptive dynamic programming using fuzzy neural networks for cloud services," IEEE Access, vol. 3, pp. 22602269, 2015.

[11] Y. Chen, J. Huang, C. Lin, and X. Shen, "Multi-objective service composition with QoS dependencies," IEEE Transactions on Cloud Computing, vol. 7, no. 2, pp. 537-552, 2019.

[12] X. Luo, J. Liu, D. Zhang, and X. Chang, "A large-scale web QoS prediction scheme for the Industrial Internet of Things based on a kernel machine learning algorithm," Computer Networks, vol. 101, pp. 81-89, 2016.

[13] J. Parejo, S. Segura, P. Fernandez, and A. Ruiz-Cortés, "QoSaware web services composition using GRASP with path relinking," Expert Systems with Applications, vol. 41, no. 9, pp. 4211-4223, 2014.

[14] C. Mao, J. Chen, D. Towey, J. Chen, and X. Xie, "Search-based QoS ranking prediction for web services in cloud environments," Future Generation Computer Systems, vol. 50, pp. 111-126, 2015.

[15] Z. ZhiJun Ding, J. JunJun Liu, Y. YouQing Sun, C. ChangJun Jiang, and M. MengChu Zhou, "A transaction and QoS-aware service selection approach based on genetic algorithm," IEEE Transactions on Systems, Man, and Cybernetics: Systems, vol. 45, no. 7, pp. 1035-1046, 2015.

[16] N. Temglit, A. Chibani, K. Djouani, and M. A. Nacer, "Distributed approach for QoS service selection in web of objects," Procedia Computer Science, vol. 83, pp. 1170-1175, 2016.

[17] G. Shen-jun, C. Jie, T. Hao-cheng, X. Ping, and Y. Yun, "An agent-based distributed QoS multicast routing algorithm," Physics Procedia, vol. 24, pp. 1951-1958, 2012.

[18] A. F. M. Huang, C.-W. Lan, and S. J. H. Yang, "An optimal QoS-based web service selection scheme," Information Sciences, vol. 179, no. 19, pp. 3309-3322, 2009.

[19] W. Lo, J. Yin, Y. Li, and Z. Wu, "Efficient web service QoS prediction using local neighborhood matrix factorization," Engineering Applications of Artificial Intelligence, vol. 38, pp. 14-23, 2015. 